\title{
Plasmonic properties of gold-palladium core-shell nanorods
}

\author{
Kitsakorn Locharoenrat and Pattareeya Damrongsak \\ Department of Physics, Faculty of Science, King Mongkut's Institute of Technology \\ Ladkrabang, Bangkok 10520, Thailand, klkitsak@kmitl.ac.th
}

Received: 23.04 .2015

\begin{abstract}
We focus on the plasmonic properties of palladium-coated gold nanorods. Two characteristic plasmon bands of those nanorods have been detected in the optical absorption. One of them, which is located at about $525 \mathrm{~nm}$, is associated with electron oscillations along the transverse direction. This band does not depend on the palladium-shell thickness and the dielectric susceptibility of the surrounding medium. The other band, at about $800-900 \mathrm{~nm}$, is associated with electron oscillations along the longitudinal direction. It exhibits a clear shift when the palladium-shell thickness and the dielectric surrounding are changed. Our results point to a novel possible way for tuning photo-catalytic ability of the nanorods and their possible use in biological/chemical sensors.
\end{abstract}

Keywords: metals, plasmons, nanomaterials, nanorods

PACS: 73.20.Mf, 78.67.Qa, 81.10.Dn

UDC: $535.34+535.331$

\section{Introduction}

Bimetallic nanoparticles can offer additional degrees of freedom when compared with the pure elemental particles, by altering physical properties of the latter. This can enable a wide range of applications, e.g. catalysis technologies [1-6] or optical devices [7-11]. Like bimetallic nanoparticles, applications of bimetallic nanorods have served as a catalyst of one of the most active areas of the nanoscience. Bimetallic nanorods having large surface-to-volume ratios are among the nanomaterials used to improve selectivity and rate of metal-catalyzed reactions. Moreover, the bimetallic nanorods employed as nanocatalysts can provide a way for utilizing smaller amounts of expensive catalyst materials, by using less expensive metals as core materials. On the other hand, the structured core-shell nanocatalysts retain an efficient coupling surfaceplasmon resonance. They can, for instance, act as built-in sensing components, which are able to signal the exposure to biological agents and toxic chemicals before the doses of the latter become harmful.

So far, the bimetallic nanorods have been used mainly in alloy or core-shell forms, relying significantly on the synthesis conditions, miscibility and the kinetics of reduction of metal ions. In this work, we study the plasmonic properties of the core-shell particles where palladium-wrapped gold nanorods have been obtained with various palladium-shell thicknesses and surrounding media. Like platinum, palladium is unique for its catalytic properties. It is expected to be a highly useful industrial catalyst for producing hydrogen from methane, reducing automobile pollutant gases, and even in direct methanol-fuel cells.

Gold has also attracted much attention as a potential core for bimetallic core-shell nanorods. The reasons are its strong optical absorption in the visible region [12, 13] and good catalytic properties [14-16], which reveal clear size and shape dependences. Furthermore, the use of gold 
for the catalysis, instead of the other metals commonly employed for that aim, is more viable economically. In this respect our experimental results may prove to be very useful for the solar energy conversion by plasmonic luminescent solar concentrators. Moreover, the benefits from the plasmonic effect can be applied to develop metal-enhanced fluorescence via fluorescent dye-doped $\mathrm{Au} @ \mathrm{Pd}$ thin films. This will be the subject of our forthcoming article.

The impact of the present work concerns the two aspects. First, we have measured the exact values of the metal-component concentrations. Since the metal mass in the solution cannot be extracted from its precursors, one needs inductively coupled plasma-mass spectrometry examinations of the products obtained finally, especially in the case of our plasmonic investigations. We have used the mentioned spectrometric technique to measure the exact metal concentrations. Notice that some of the studies (see Refs. [17, 18]) provide only approximate estimations issuing from the metal sources and the conversion efficiency. Although these rough concentration estimations are of the same order of magnitude, they can vary notably from the exact values. Second, we have built a simpler, lower-cost spectrometer, as compared with its commercial counterparts.

\section{Experimental}

A gold core was prepared from the solution of cetyltrimethylammonium bromide, using a seedmediated growth technique [19]. Palladium chloride components of different concentrations were then added to the gold core in order to form a palladium shell of varying thickness. The exact concentrations were examined with the inductively coupled plasma-mass spectrometry, which is usually used for determining the exact amounts of metals. The gold-core sizes and the palladiumshell thicknesses were characterized using a transmission electron microscopy. The samples were centrifuged and redispersed in deionized water before the optical absorption measurements.

The absorption spectra (in arbitrary units) of the gold-palladium core-shell nanorods with different weight ratios of palladium and gold ( $\mathrm{Pd}: \mathrm{Au}=0.20: 1,0.10: 1,0.05: 1$ and 0:1) were measured in the wavelength region $400-900 \mathrm{~nm}$, using a home-made spectrometer equipped with polarizing accessories, a xenon lamp Hamamatsu L2273, and a photomultiplier Hamamatsu R585 (see Fig. 1). We used two monochromators in a series, which functioned as a double filter for rejecting any stray light. The exit slit of the first monochromator was the entrance slit for the second one. The absolute values of sample absorptions were obtained by normalizing the sample absorption response corresponding to plasmon resonances to that of a quartz reference plate.

Finally, the optical absorptions of a set of palladium-coated gold nanorods placed in the liquids with different dielectric constants (see Table 1) were studied. The plasmon modes were also detected for the reference cases of refined ethanol and toluene. The experimental data was presented in terms of dependence of the plasmon-peak wavelength on the refractive index.

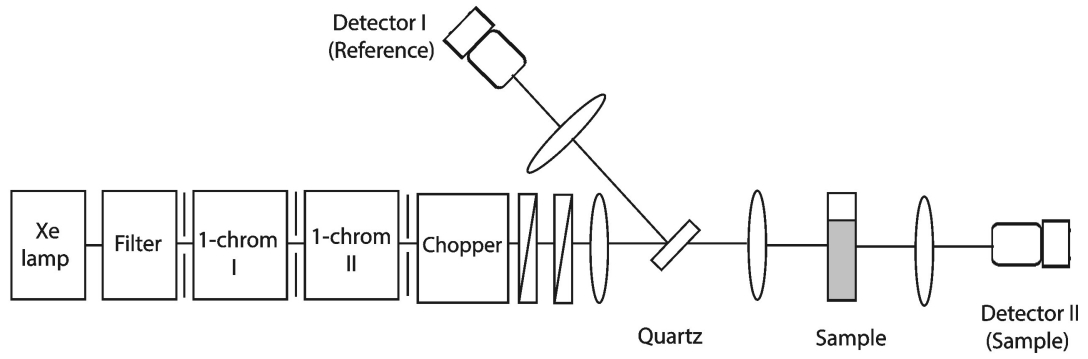

Fig. 1. Schematic diagram of our optical setup. '1-Chrom I' and '1-Chrom II' are two monochromators (see the text).

Ukr. J. Phys. Opt. 2015, Volume 16, Issue 3 
Table 1. Chemical substances used in this work and their refractive indices

\begin{tabular}{|c|c|c|}
\hline Ethanol fraction (\%) & Toluene fraction (\%) & Refractive index \\
\hline 0 & 0 & 1.33 \\
\hline 100 & 0 & 1.36 \\
\hline 67 & 33 & 1.41 \\
\hline 50 & 50 & 1.43 \\
\hline 33 & 67 & 1.45 \\
\hline 0 & 100 & 1.49 \\
\hline
\end{tabular}

\section{Results and discussion}

The transmission electron microscopy images displayed in Fig. 2a and Fig. 2b show uniform gold nanorods and uniform palladium-coated gold nanorods, respectively. The short axis $\mathrm{b}$ and the long axis a of the gold nanorod core are $40 \pm 3$ and $96 \pm 6 \mathrm{~nm}$, respectively. The aspect ratio a/b is therefore $2.4 \pm 2$. The thickness of the coating palladium shell is equal to $3.0 \pm 0.2 \mathrm{~nm}$.

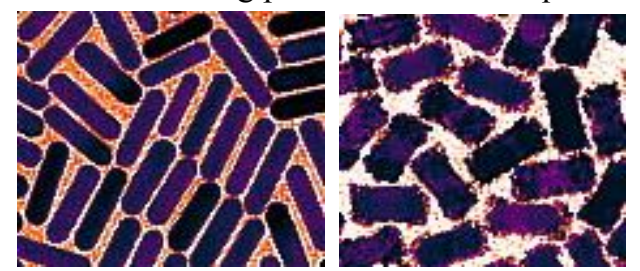

Fig. 2. Transmission electron microscopy images of gold (left panel) and palladium-coated gold (right panel) nanorods.

The optical absorption spectra measured for the palladium-coated gold nanorods with the $\mathrm{Pd} / \mathrm{Au}$ weight ratios $0,0.05,0.10$ and 0.20 are shown in Fig. 3. Two main plasmon peaks can be detected for all of our samples. The first is linked to electron oscillations perpendicular to the long axis of the rod (a so-called transverse plasmon band), and the second is associated with conduction-electron oscillations parallel to the long axis of the rod (a so-called longitudinal plasmon band). Of those two modes, the transverse oscillations of electrons give rise to the absorption band located at the shorter-wavelength side (at about $525 \mathrm{~nm}$ ), which corresponds to nanospheres. That location of the resonance is independent of the palladium-shell thickness and the changing $\mathrm{Pd} / \mathrm{Au}$ weight ratio because the short axis a is dominant for the spherical-like segments. The resonance shows no detectable shift when the $\mathrm{Pd} / \mathrm{Au}$ weight ratio increases from 0.05 to 0.20 . This result can be explained with the approach developed by Sandrock et al. [20] suggesting that the light polarization is weakly affected by the centrosymmetric structures having the aspect ratio $\mathrm{a} / \mathrm{b}=1$.

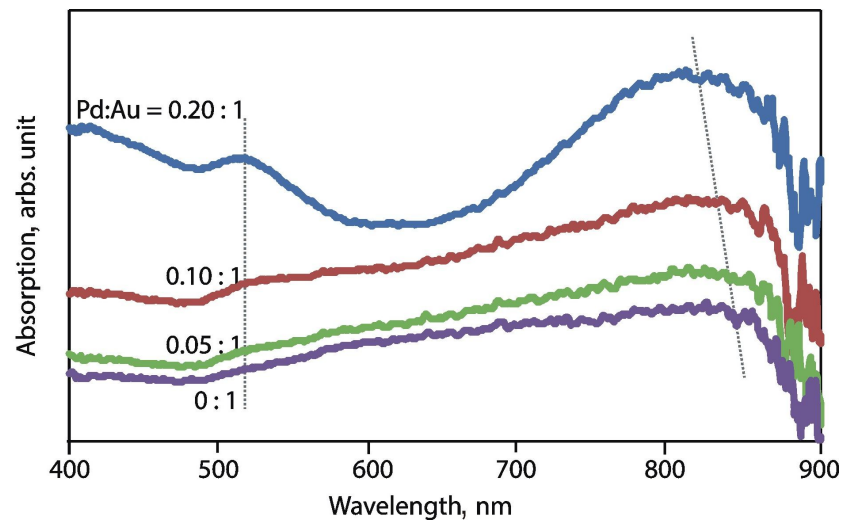

Fig. 3. Absorption spectra of palladiumcoated gold nanorods with different $\mathrm{Pd} / \mathrm{Au}$ weight ratios. Left and right peaks correspond respectively to transverse and longitudinal plasmon bands. Each sample is immersed in deionized water. 
On the other hand, the longitudinal oscillations of electrons result in the band located in the longer-wavelength part of the spectrum, because the long axis b is dominant for the case of rodlike segments. The peak position depends on the palladium-shell thickness, thus suggesting that the effect of gold core on the plasmonic sensitivity is completely screened when the palladiumshell thickness becomes larger. As a consequence, increasing thickness of the palladium shell imposes a blue shift of the plasmon absorption maximum. This longitudinal plasmon band corresponds to the optical response of the pure palladium nanorods, due to less negative values of the real part of the permittivity of palladium as compared to gold [21]. For instance, the absorption band shifts from 860 to $820 \mathrm{~nm}$ when the $\mathrm{Pd} / \mathrm{Au}$ weight ratio increases from 0.05 to 0.20 . This result can also be explained using the results by Sandrock et al. [20], which suppose that the light polarization is strongly affected by the noncentrosymmetric structures having the aspect ratio $\mathrm{a} / \mathrm{b} \neq 1$. In other words, by varying the $\mathrm{Pd} / \mathrm{Au}$ ratio, one can adjust the blue shift of the plasmon band to be anywhere beginning from 860 and ending at $820 \mathrm{~nm}$.

To study dependence of the plasmon peak on the refractive index of the surrounding medium, we have dispersed the palladium-coated gold nanorods into different organic solvents (The relevant refractive indices are displayed in Table 1). Fig. 4 shows the peak positions of the plasmon resonance for different Pd:Au nanorods immersed into different organic dielectric media. The peak location for the transverse plasmon band is independent of the dielectric medium. This implies that the absorption peak located at $525 \mathrm{~nm}$ does not change when the refractive index of the medium increases from 1.33 to 1.49 (not shown in Fig. 4).

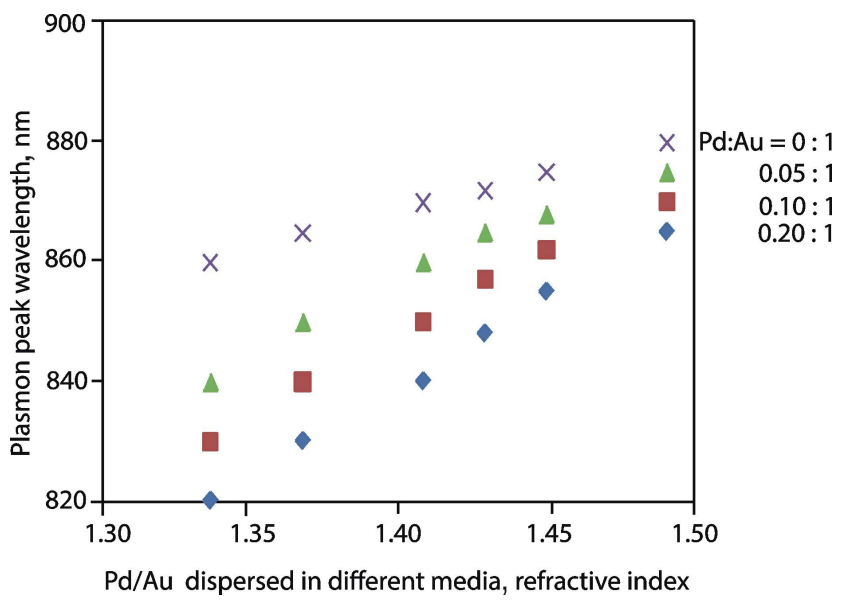

Fig. 4. Plasmon peak wavelengths corresponding to the longitudinal plasmon mode for the $\mathrm{Pd} / \mathrm{Au}$ nanorods immersed in different dielectric media (see Table 1).

By contrast, due to recognizable dependence of the plasmonic sensitivity for the longitudinal mode on the palladium-shell thickness, the palladium-coated gold nanorods with different palladium-shell thicknesses give rise to distinct plasmon-peak wavelengths even in the same surrounding medium. Namely, the plasmon peak wavelengths for the nanorods having the ratios $\mathrm{Pd}: \mathrm{Au}=0.20: 1,0.10: 1,0.05: 1$ and $0: 1$, which are immersed in deionized water $(\mathrm{n}=1.33)$, are respectively equal to $820,830,840$ and $860 \mathrm{~nm}$. Then, by varying the $\mathrm{Pd} / \mathrm{Au}$ ratio for the case of the same medium, one can adjust the plasmon-band shift to be anywhere from 820 to $860 \mathrm{~nm}$.

Furthermore, the peak position of the longitudinal plasmon band with a given palladium-shell thickness shows evident red shift with increasing refractive index. Namely, the plasmon peak shifts for the cases of $\mathrm{Pd}: \mathrm{Au}=0.20: 1,0.10: 1,0.05: 1$ and $0: 1$ are respectively 820-860, 830-867, 840-874 and 860-880 $\mathrm{nm}$, when each sample is immersed in the medium with different refractive index (1.33-1.49). The shift value depends linearly on the refractive index. The host media with 
higher refractive indices (i.e., higher dielectric constants) are effectively more polarizable and thus couple more readily with the surface plasmon electrons. The energy needed to collectively excite electrons is then reduced. In this case the maximum of the plasmon absorbance is shifted towards the red side of spectrum.

There are two possible origins of the observed spectral changes associated with the electron oscillations in our samples. First, there is a change in the electromagnetic field gradient normal and/or parallel to the sample plane, due to the plasmon excitation. Second, as seen from Fig. 2, an electro-static lightning-rod effect via geometric singularity of sharply pointed structures may result in enhancement of the electric field. Numerical investigations of the local electromagnetic fields with the finite-difference time-domain technique are now in progress. They can unravel the nature of the lightning-rod effect and plasmon modes enhancing in our samples. The more detailed results will be presented in our forthcoming article.

In summary, both the presence of the palladium shells deposited onto the Au-core nanorods and the changes in the refractive index of the surrounding dielectric medium are marked by a change in the sample colour and a shift in the plasmon-peak position proportional to the changes in the electromagnetic field located around the nanorod surfaces. The UV-Vis-NIR absorption spectra clearly point to both short- and long-wavelength plasmon bands, which are attributed respectively to the transverse and longitudinal plasmon modes of the rods. Under controlled changes in the palladium-shell thickness and the refractive index of the surrounding dielectric medium, the longer-wavelength plasmon band reveals a shift, whereas the shorter-wavelength band remains invariable. Since the palladium-coated gold nanorods have an inherent photo-sensing ability, they should prove a very desirable material for the photo-catalysis and for the surface plasmon resonance-based sensors.

\section{Conclusions}

We have described two observations regarding the optical properties of the gold-palladium coreshell nanorods. The nanorods reveal a unique optical response, with the two characteristic plasmon resonance bands present in the optical absorption. Unlike the resonant peak from the transverse plasmon band, the peak linked with the longitudinal plasmon band excitation shows a remarkable shift when one changes the palladium-shell thickness and the dielectric medium in a controllable manner. A blue shift of the localized longitudinal surface plasmon resonance in the UV-Vis-NIR absorption spectrum is observed if more palladium atoms are added in order to form a shell on the gold core. This allows one to alter the plasmon resonance of the palladium-coated gold nanorods.

On the other hand, since high enough refractive indices of the organic solvents can screen the incident electromagnetic field, the localized longitudinal surface plasmon resonance becomes redshifted with increasing refractive index of the surrounding medium. Due to excellent reproduction of the absorption response and the intense plasmon peaks, the palladium-coated gold nanorods can serve as a superior candidate for chemical/biological sensing and photo-catalysis.

\section{Acknowledgment}

This work was supported by the King Mongkut's Institute of Technology Ladkrabang (Bangkok 10520, Thailand) under the Grant 39/2013.

\section{References}

1. Liu H, Ye F, Yao Q, Cao H, Xie J and Yang J, 2014. Stellated Ag-Pt bimetallic nanoparticles: An effective platform for catalytic activity tuning. Sci. Rep. 4: 3669. 
2. Hu G, Nitze F, Gracia-Espino E, Ma J, Barzegar HR, Sharifi T, Jia X, Shchukarev A, Lu L, Ma C and Wagberg T, 2014. Small palladium islands embedded in palladium-tungsten bimetallic nanoparticles form catalytic hotspots for oxygen reduction. Nature Commun. 5: 5253 .

3. Liu W J, Qian T T and Jiang H, 2014. Bimetallic Fe nanoparticles: Recent advances in synthesis and application in catalytic elimination of environmental pollutants. Chem. Eng. J. 236: $448-463$.

4. Konig R Y G, Schwarze M, Schomacker R and Stubenrauch C, 2014. Catalytic activity of mono- and bi-metallic nanoparticles synthesized via microemulsions. Catalysts. 4: 256-275.

5. Rao V K and Radhakrishnan T P, 2013. Hollow bimetallic nanoparticles generated in situ inside a polymer thin film: Fabrication and catalytic application of silver-palladium-poly (vinyl alcohol). J. Mater. Chem. A. 1: 13612-13618.

6. Zhang H, Haba M, Okumura M, Akita T, Hashimoto S and Toshima N, 2013. Novel formation of $\mathrm{Ag} / \mathrm{Au}$ bimetallic nanoparticles by physical mixture of monometallic nanoparticles in dispersions and their application to catalysts for aerobic glucose oxidation. Langmuir. 29: 10330-10339.

7. Boote B W, Byun H and Kim J H, 2014. Silver-gold bimetallic nanoparticles and their applications as optical materials. J. Nanosci. Nanotechnol. 14: 1563-1577.

8. Adekoya J A, Dare E O, Mesubi M A and Revaprasadu N, 2014. Synthesis and characterization of optically active fractal seed mediated silver nickel bimetallic nanoparticles. J. Mater. 2014: 184216.

9. Perez J L J, Fuentes R G, Ramirez J F S, Vidal O U G, Tellez-Sanchez D E, Pacheco Z N C, Orea A C and Garcia J A F, 2013. Nonlinear coefficient determination of Au/Pd bimetallic nanoparticles using Z-scan. Adv. Nanoparticles. 2: 223-228.

10. Arquilliere P P, Santini C, Haumesser P H and Aouine M, 2011. Synthesis of copper and copper-ruthenium nanoparticles in ionic liquids for the metallization of advanced interconnect structures. ECS Transac. 35: 11-16.

11. Sachan R, Yadavali S, Shirato N, Krishna H, Ramos V, Duscher G, Pennycook S J, Gangopadhyay A K, Garcia H and Kalyanaraman R, 2012. Self-organized bimetallic Ag-Co nanoparticles with tunable localized surface plasmons showing high environmental stability and sensitivity. Nanotechnol. 23: 275604.

12. Hu M, Chen J, Li Z Y, Au L, Hartland G V, Li X, Marquez M and Xia Y, 2006. Gold nanostructures: Engineering their plasmonic properties for biomedical applications. Chem. Soc. Rev. 35: 1084-1094.

13. Dykman L A and Khlebstov N G, 2011. Gold nanoparticles in biology and medicine: Recent advances and prospects. Acta Nature. 3: 34-55.

14. Gates B C, 2013. Supported gold catalysts: New properties offered by nanometer and subnanometer structures. Chem. Commun. 49: 7876-7877.

15. Zhou X, Xu W, Liu G, Panda D and Chen P, 2010. Size-dependent catalytic activity and dynamics of gold nanoparticles at the single-molecule level. J. Amer. Chem. Soc. 132: 138146.

16. Zhu Y, Jin R and Sun Y, 2011. Atomically monodisperse gold nanoclusters catalysts with precise core-shell structure. Catalysts. 1: 3-17.

17. Ruibin J, 2013. Plasmonic properties of bimetallic nanostructures and their applications in hydrogen sensing and chemical reactions. Dissert. Abs. Inter. 75-06: 1-176.

Ukr. J. Phys. Opt. 2015, Volume 16, Issue 3 
18. Hao J and Hui W, 2014. Controlled overgrowth of Pd on Au nanorods. CrystEngComm 16: 9469-9477.

19. Pradeep T, A textbook of nanoscience and nanotechnology. New York: McGraw Hill (2012).

20. Sandrock M L, Geiger F M and Foss C A, 1999. Synthesis and second-harmonic generation studies of noncentrosymmetric gold nanostructures. J. Phys. Chem. B. 103: 2668-2673.

21. Palik E D, Handbook of optical constants of solids. New York: Academic Press (1991).

Kitsakorn Locharoenrat and Pattareeya Damrongsak. 2015. Plasmonic properties of goldpalladium core-shell nanorods. Ukr.J.Phys.Opt. 16: 120 - 126.

Анотація. У роботі досліджено плазмонні властивості золотих наностержнів, покритих паладієм. У спектрах їхнього оптичного поглинання виявлено дві характерні плазмонні смуги. Одна з них, розташована в околі 525 нм, пов'язана з електронними коливаннями вздовж поперечного напрямку стержнів. Ї̈ положення не залежить від товщини паладієвої оболонки та діелектричної сталої оточуючого середовища. Інша, розташована в околі 800-900 нм, асочіюється з електронними коливаннями в поздовжньому напрямку. Вона зазнає зсуву зі зміною товщини паладієвої оболонки $і$ показника заломлення оточуючого середовища. Наші дослідження вказують на новий можливий шлях керування фотокаталітичною здатністю наностержнів $i$ перспективи їхнього використання 6 біолого-хімічних сенсорах. 